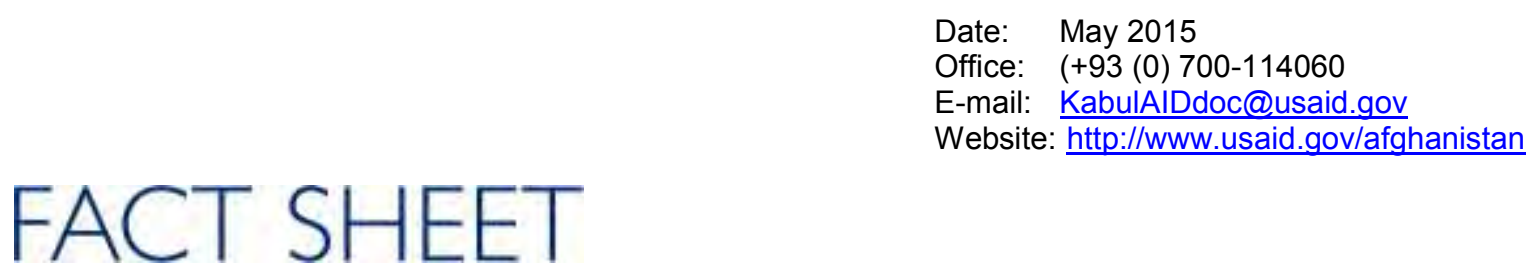

\title{
Improved Health for Afghan Mothers and Children
}

\section{OVERVIEW}

In 2002, Afghanistan had one of the highest rates of maternal and child mortality in the world due to a lack of basic health care, equipment and facilities. As a result of the Afghan Government and development partners' efforts to improve health services and public health policy, these rates are declining but remain among the highest in the region.

Afghanistan's current maternal mortality rate is estimated at 327 per 100,000 . This means that for every 1,000 live births, an estimated three women die during pregnancy, in childbirth or in the two months after delivery. (In the U.S.,18.5 mothers died per 100,000 births in 2013.) Despite a decrease in the child mortality rate, an estimated one out of 10 Afghan children will die before their fifth birthday. Most causes of maternal and child mortality in Afghanistan are preventable. Even though Afghanistan has made tremendous accomplishments, much work is still needed. USAID remains committed to building Afghanistan's health sector to improve maternal and child health.

\section{CURRENT ACTIVITIES}

While maternal mortality remains high in Afghanistan, USAID partners and programs are reducing pregnancy-related deaths. USAID is increasing access to higher quality and more skilled maternal care (pregnancy, birth, post-partum), addressing major causes of maternal mortality, and scaling up highimpact interventions. USAID is:

- Increasing training in basic and emergency obstetric and neonatal care.

- Strengthening the quality of family planning and reproductive health services, such as contraceptive use to encourage birth spacing, and promoting respectful care and counseling.

- Providing medication at the community level and in clinics to prevent postpartum hemorrhage, a chief cause of maternal death.

- Expanding community-based services through the existing community health worker network to increase household delivery of lifesaving services, such as misprostol distribution and promoting birth preparedness.

- Distributing iron-supplements to adolescent girls and pregnant women to prevent iron-deficiency.

- Training midwives to provide maternal health care at the community level

- Supporting the first Demographic Health Survey in Afghanistan to collect rigorous demographic health data at the national and provincial level to inform changes in maternal and child health programs and policies

Progress in child survival and disease control are among USAID's biggest priorities. Children under five are saved each year through USAID-funded interventions. USAID continues to:

- Support management of childhood illness with special emphasis on diarrhea treatment, one of the leading causes of under-five deaths in Afghanistan.

- Train doctors and midwives in all public health facilities on newborn resuscitation.

- Strengthen the Ministry of Public Health (MoPH), NGOs and professional associations such as the Afghanistan Midwife Association to provide and oversee newborn care services at health clinics. 


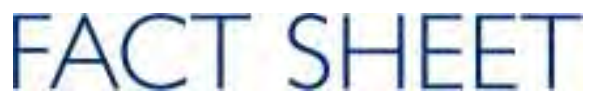

- Promote infant and child feeding practices at the community level, especially breastfeeding until six months, and increase food variety and frequency of feeding as the child grows.

- Increase the ability of MoPH and NGOs to deliver basic health services to improve nutrition status, such as scaling up vitamin A supplementation.

- Promote and improve good hygiene and sanitation practices (hand washing, waste disposal, and proper food preparation and storage).

- Fortify food staples through a regional nutrition initiative.

\section{ACCOMPLISHMENTS}

- Basic health services are delivered to more than 50 percent of the Afghan population.

- Through the Disease Early Warning System, 387 sites to detect infectious disease outbreaks have been established, monitoring 15 infectious diseases across Afghanistan and responding to 1,829 outbreaks. Approximately 98 percent are investigated within 48 hours of notification, reducing morbidity and mortality through early warning and response.

\section{Child Health}

- In 2014, over 469,000 children received vaccines against three infectious diseases (diphtheria, whooping cough and tetanus) by 12 months of age and over 172,000 births were delivered by trained doctor or midwives.

- Marketed more than 300,000 sachets of oral rehydration salts to prevent dehydration among children suffering from diarrhea.

\section{Maternal Health:}

- The percentage of women receiving care before birth has increased from 16 percent in 2002 to 60 percent in 2010 (a 44 percent increase).

- The percentage of women attended by a skilled provider at birth increased from 14 percent in 2002 to 52 percent in 2014.

- In 2014, 69 percent of pregnant women were immunized against tetanus toxoid.

- Supported the establishment of the professional Afghan Midwife Association, which has trained 2,300 community-midwives.

- Supported the increase of women in the health workforce. In 2002, only 25 percent of health facilities had a female health worker. As of early 2015, 85 percent of health facilities have at least one female health worker (doctor, midwife or nurse). 